Archives de sciences sociales des religions

172 | octobre-décembre

Bulletin Bibliographique

\title{
Retour sur et avec Jean-Pierre Deconchy (1934-2014)
}

Pierre Lassave

\section{(2) OpenEdition \\ 12 Journals}

Édition électronique

URL : http://journals.openedition.org/assr/27207

DOI : $10.4000 /$ assr.27207

ISSN : $1777-5825$

Éditeur

Éditions de l'EHESS

Édition imprimée

Date de publication : 1 octobre 2015

Pagination : 13-24

ISBN : 978-2-7132-2515-4

ISSN : 0335-5985

Référence électronique

Pierre Lassave, « Retour sur et avec Jean-Pierre Deconchy (1934-2014) », Archives de sciences sociales des religions [En ligne], 172 | octobre-décembre, mis en ligne le 26 avril 2018, consulté le 01 mai 2019. URL : http://journals.openedition.org/assr/27207 ; DOI : 10.4000/assr.27207

() Archives de sciences sociales des religions 


\section{Pierre Lassave}

\section{Retour sur et avec Jean-Pierre Deconchy (1934-2014)}

Dans notre livraison du premier trimestre 2015 ( $N^{\circ} 169$ ), nous avions rendu hommage à notre collègue Jean-Pierre Deconchy, professeur émérite en psychologie sociale à l'Université de Nanterre, disparu le 30 novembre 2014, qui fut membre du comité de rédaction de notre revue et responsable des tables analytiques et du bulletin des périodiques de 1969 à 1991. Nous voudrions prolonger ici doublement cet hommage. D'une part, en publiant un entretien inédit sur son parcours de recherche, notamment sa rencontre avec le Groupe de sociologie des religions (GSR) et sa revue et le souvenir qu'il en gardait peu avant sa mort brutale. D'autre part, en joignant à notre entretien un article bilan de Magdalini Dargentas sur la psychologie expérimentale des religions aujourd'hui. Ce dernier texte, normalement destiné à la rubrique des Varia, livre une synthèse de cette discipline spécialisée dont le développement doit beaucoup aux travaux novateurs de Jean-Pierre Deconchy.

L'entretien qui suit s'inscrit dans une série de témoignages vivants recueillis autour de la mémoire du Groupe de sociologie des religions et de notre revue. Une rubrique de notre site internet précise les contours de ce projet d'enquête et de réflexion sur l'histoire des catégories et des figures du savoir qui font aujourd'hui la spécificité fragile et diverse des sciences sociales du religieux (assr.revues.org). Dans cet entretien, le chercheur nous livre quelques tenants et aboutissants de son parcours : son enfance dans le milieu des ouvriers textiles du nord de la France, sa rencontre avec la foi et avec une Église missionnaire qui le forme aux humanités et au sacerdoce, ses premières observations comportementales lorsque, jeune instituteur dans une école du Liban, il découvre que les enfants lisent et dessinent de droite à gauche, dans le sens inverse de ce qu'il a vécu en France. Expérience fondatrice à divers égards, puisque le futur prêtre professeur de philosophie ne cessera depuis lors de s'intéresser aux mécanismes sociaux et mentaux qui forment les pensées et les gestes. Le récit qu'il nous livre retrace le chemin qu'il accomplit pour aller au bout de ses interrogations, en particulier jusqu'à rompre avec l'Église dont les productions imaginaires et normatives se transmuent désormais en objets d'expérimentation. 
C'est ainsi qu'il montre dans sa thèse universitaire comment les représentations enfantines du divin se trouvent systématiquement associées chez les filles à des images paternelles et chez les garçons à des images maternelles. Henri Desroche et Émile Poulat, autres clercs ayant quitté l'Église pour la science, ont accueilli le jeune chercheur dans le Groupe de sociologie des religions pour y développer cette psychologie des religions aussi prometteuse qu'inchoative. Associé en même temps au Laboratoire de psychologie sociale animé en Sorbonne par Robert Pagès, notre spécialiste multiplie les observations expérimentales avec tous les protocoles requis. Il émet par exemple l'hypothèse que la fermeté d'action de toute orthodoxie religieuse s'impose d'autant plus qu'elle doit compenser la fragilité de ses justifications rationnelles. Chemin faisant, l'assimilation du religieux à n'importe quel système idéologique ou propositionnel éloigne la perspective d'en faire un objet spécifique de la psychologie expérimentale. D’où sans doute la relative invisibilité de la psychologie expérimentale des religions par rapport aux avenues peuplées de l'interprétation psychanalytique des représentations imaginaires du religieux. C'est d'ailleurs là que le bilan dressé par Dargentas s'avère utile pour s'enquérir de ce qui peut être retenu de l'héritage de son collègue Deconchy aujourd'hui.

Mais l'entretien transcrit, dont on a volontairement gardé la forme vivante de l'oral, nous parle aussi d'une époque d'engagements cumulés ou successifs de soi, tant dans une foi rédemptrice d'un monde à peine sorti de la Shoah que dans une science comme nouvelle forme de sagesse consciente de tout ce qui dans l'être est agi à son insu. L'évocation de ce temps de promesses, mais aussi d'inventions ou de bricolages d'idées, de structures, de paradigmes et de protocoles expérimentaux n'a rien de nostalgique. Le recul de l'âge permet au contraire d'accuser ses traits non sans quelque dose d'humour sur le pittoresque des situations : par exemple, les missionnaires de la science des religions emmenés par leur chef de file Henri Desroche qui commande effrontément une bouillabaisse dans un restaurant du Québec ; ou bien les séances de dégustation du « roudoudou " - décoction de calice de rose - rite de détente dominicale plaisamment réglé par l'austère Jean Séguy. Ou enfin ces textes bibliques expressément grevés de contresens pour les besoins d'un test de vérité que les pasteurs en exercice trouvent finalement plus pertinents que les textes initiaux. Mais la mémoire ne retient pas moins les tensions interpersonnelles et épistémiques d'un milieu solidaire car quelque peu marginal - la sociologie des religions -, tensions ou clivages qui rappellent le devenir incertain des sciences sociales du religieux. Il en va ainsi de ce paradoxe souligné par notre interlocuteur qui veut que la diversification nécessaire des terrains, des disciplines et des méthodes aille à l'encontre de la conceptualisation de ce qui se dit et se fait religieux.

Le 3 juillet dernier, l'université Paris-Ouest-Nanterre a tenu une "Journée d'hommage au Professeur Jean-Pierre Deconchy » en son Laboratoire de psychologie sociale. C'est dire que depuis les années 1980, notre collègue a trouvé, après 
l'Église puis le GSR, une troisième famille d'accueil. Les actes à venir de ce riche moment d'échange montreront sans doute que le professeur y a fait souche. Pas seulement à Nanterre d'ailleurs si l'on en juge par ses collègues italiens, grecs, marocains, canadiens ou africains. Ses élèves devenus à leur tour professeurs font désormais passer dans leur enseignement et leur recherche l'esprit frondeur, inventif et sans cesse interrogatif de leur maître. À écouter les multiples témoignages sur ses travaux allant de la lecture par la droite ou la gauche (exhumés et traduits récemment en anglais) jusqu'aux " animaux surnaturés ", en passant par l'orthodoxie religieuse, on ne peut qu'être frappé par une sorte de constante cognitive et même pratique consistant à déceler toujours, sinon exorciser, la part d'inexplicable dans l'expérience humaine. La tension entre le schème de la rationalisation logique et celui de la narration fantastique qui oscille suivant les situations culturelles des locuteurs pour rendre compte des "phénomènes inexplicables de prime abord »n'a donc pas fini d'offrir ses énigmes à quiconque s'interroge sur les chassés-croisés du croire et du savoir.

L'ancrage final de notre collègue dans ce milieu vivant de la psychologie expérimentale atteste une fois de plus que le GSR n'a pas seulement été un des canaux historiques de la légitimation de l'objet religieux dans les sciences sociales mais qu'il a été aussi un incubateur de parcours de recherche qui le dépassent. JeanPierre Deconchy est ainsi passé de la psychologie sociale des religions à la psychologie expérimentale des idéologies, à l'instar d'un Henri Desroche passé de la sociologie des messianismes à celle de la coopération ou d'un François-André Isambert passé de la sociologie de la sécularisation à celle de la bioéthique. Mais place ici à la parole de notre témoin. Ses propos ont été recueillis et transcrits par moi-même en février 2014 puis validés par leur auteur dans la perspective d'une éventuelle publication. Les notes en bas de page et une bibliographie succincte précisent certaines références.

Pierre LASSAVE

Centre d'études en sciences sociales du religieux (CéSor, EHESS-CNRS) lassave@ehess.fr

$* * *$

Nous allons si vous le voulez bien commencer par votre rencontre avec le GSR, comment cela s'est-il passé pour vous?

Oui, alors en ce qui me concerne, il faut que je dise d'abord toute ma reconnaissance pour ce groupe, ne serait-ce que statutairement, car ce sont ses membres fondateurs qui m'ont mis le pied à l'étrier. Jacques Maître a ainsi essayé de me faire entrer en sociologie au CNRS, mais j'ai échoué par trois fois, et ils m'ont orienté vers Robert Pagès en psychologie sociale, où ça a marché du premier coup. La deuxième chose à dire, c'est que je garde un très bon souvenir de ce 
groupe qui était à la fois cocasse, puissant et pittoresque. Qui n'a pas entendu Séguy racontant son mai 68 , où il se hâtait d'écrire calfeutré chez lui les dernières lignes de sa thèse à la lumière des rues enflammées par la révolte étudiante ! Qui n'a pas vécu ce moment incroyable où Desroche entre dans un restaurant de Sherbrooke pour demander une bouillabaisse ? On lui a quand même apporté un plat de poisson qui l'a mis dans une rage folle car cette bouillabaisse québécoise n'avait rien d' "orthodoxe" disait-il dans un éclat de rire! Bien alors comment en suis-je arrivé au GSR... Il faut d'abord dire que j'étais ecclésiastique à l'origine...

\section{Ecclésiastique, c'est-à-dire?}

Eh bien, j'étais prêtre au diocèse de Lille. Et si vous voulez qu'on remonte un peu plus loin, j'ai été chassé du lycée en troisième pour incapacité intellectuelle. Puis j'ai suivi une école professionnelle de textile. J'ai travaillé dans le triage de laine, et c'est dans cette période que j'ai rencontré la révélation chrétienne. Je suis alors entré au séminaire des vocations tardives d'Hazebrouck ${ }^{1}$. De là, j’ai suivi la filière de séminaire académique. En 1962, j’ai exercé un an comme professeur de philo dans un très bon collège privé de Roubaix. Pour l'anecdote, un de mes meilleurs élèves était l'actuel président de la commission des lois du Sénat, Jean-Pierre Sueur. Il faut que je dise que je suis issu d'un milieu plutôt prolétaire mais non misérable : ma mère était "bâcleuse " dans les filatures (ouvrière de basse qualification dans l'industrie textile), seule avec trois enfants, et mon père était tout petit militaire, un milieu non politisé, donc fatalement à droite... Ce qui s'est trouvé a été alors la chance de ma vie : l'ai-je demandé ou me l'a-t-on proposé ? Je ne sais plus, mais pendant la guerre d'Algérie, j’ai été affecté durant deux ans et demi comme instituteur dans une école à Tripoli d'Asie au Liban, au titre de détaché militaire. C'était un accord entre le séminaire et l'armée. Et c'est là que j'ai commencé à faire des études de philosophie et en même temps à m'intéresser à des questions de psychologie. J'ai ainsi engagé une observation sur la manière dont les élèves d'origine arabe lisaient le français en commençant de la droite vers la gauche.

\section{Et en quelle année avez-vous été ordonné?}

Ce devait être en 1961, je crois, et si vous voulez boucler la boucle, je suis sorti de l'Église en 1969-70, sans aucun fracas ni drame interne. Ça s'est passé tout simplement parce que j'avais perdu la foi, je ne croyais plus en Dieu et je devais en tirer les conséquences. Je n'aurais pu assumer la violence d'un mensonge ou d'un porte-à-faux aussi difficile à vivre. Je n'ai jamais eu à me plaindre

1. Petit séminaire Saint François d'Assise avec sa section des vocations tardives fondée en 1908 qui avait pour but de préparer au Grand séminaire les enfants qui selon le règlement de l'établissement " offraient des garanties sérieuses de vocation sacerdotale ». 
de pressions à mon encontre au sein de l'Église comme cela s'est passé pour nombre d'intellectuels avec lesquels je me suis retrouvé par la suite. Voilà pour ce chapitre.

\section{Et donc vous étiez encore prêtre lorsque vous avez intégré le GSR ?}

Lorsque je suis revenu du Liban à la fin des années 1950, j’ai eu dans l'idée de poursuivre les études de psychologie et cela m'a amené dans le cadre de la préparation d'une thèse de théologie à une recherche sur l'idée de Dieu chez l'enfant. C'était un secteur tout nouveau de la recherche, sans perspective catéchétique ni missionnaire. Il s'agissait d'étudier les associations libres autour du mot Dieu chez 8000 enfants catholiques scolarisés dans les diocèses de Lille et de Dunkerque. Une thèse furieusement positiviste. J'ai fait à ce moment-là la connaissance d'un jésuite belge qui s'appelait André Godin ${ }^{2}$. C'était également un chercheur en psychologie religieuse comme on l'appelait à l'époque, et ce père m'a invité à participer à une réunion à Paris sur le sujet. Et donc mon premier contact avec les gens du GSR a dû se faire à l'occasion de cette réunion de travail qui s'est tenue en 1962 chez des dominicains et où j'ai rencontré Jacques Maître qui avait fait une brillante démonstration à partir de l'analyse hiérarchique des données en matière d'attitudes religieuses. Le contact entre lui et moi s'est bien passé et c'est ensuite grâce à lui que j'ai rencontré le GSR.

Puis j'ai obtenu du diocèse de Lille de venir à Paris comme aumônier de jeunes filles plus que rangées. Et j'ai trouvé alors, grâce à Maître, une petite vacation au Centre d'études sociologiques. C'est en fréquentant le milieu que j'ai présenté ensuite ma candidature comme chercheur en sociologie. Elle m'a été refusée comme je l'ai dit et c'est après que j'ai été recruté en 1966 dans la section de psychologie du CNRS. À l'époque, nombre d'ecclésiastiques se tournant vers les sciences humaines choisissaient la sociologie de préférence. C'est un peu lié à la vocation pastorale ou missionnaire. Et d'ailleurs, les résistances de l'intérieur du CNRS à ce type de profil ne manquaient pas. Je pense à Odile Benoit-Guilbot, syndicaliste et communiste, qui avait ce profil en horreur et qui disait que les prêtres préféraient la sociologie parce que c'était plus facile! Il est clair que mon dossier relevait plus de la psychologie que de la sociologie. C'est donc Maître qui m'a mis en rapport avec Robert Pagès ${ }^{3}$ qui se trouvait dans un laboratoire de psychologie sociale de la Sorbonne, un véritable repère de gauchistes hauts en couleur, un lieu foisonnant, inorganisé, mais libérateur.

2. André Godin (1915-1997) fut professeur de psychologie pastorale au Centre Lumen Vitae à Bruxelles. Il est l'auteur d'essais de référence sur la psychologie des vocations et de l'expérience religieuses.

3. Robert Pagès (1919-2007), ancien résistant, élève de Georges Canguilhem, psychologue devenu directeur de recherche au CNRS, a dirigé de 1952 à 1988 le Laboratoire de psychologie sociale fondé à la Sorbonne par Daniel Lagache. 
J'ai donc été en 1966 attaché de recherche dans ce laboratoire. Ma thèse sur la structure génétique de l'idée de Dieu chez les enfants a été codirigée par Poulat et Pagès et soutenue en 1967 à l'École pratique des hautes études (VI ${ }^{\mathrm{e}}$ section) ${ }^{4}$. Puis d'attaché, je suis devenu chargé de recherche, puis maître de recherche, puis un poste de professeur à Nanterre s'est dessiné. J'ai soutenu ma thèse d'État en 1975 sur le rapport entre sciences humaines et orthodoxie religieuse, sous la direction de Jean Maisonneuve ${ }^{5}$, contre l'avis d'ailleurs de mon directeur de laboratoire. Et à partir de 1981, j'ai suivi une carrière tout à fait classique d'enseignantchercheur, à l'époque où la recherche s'intégrait dans l'enseignement supérieur. Je me souviens que lors de mon recrutement quelqu'un a dit : il est chercheur, il reste maintenant à démontrer qu'il sera un bon enseignant! Maisonneuve parlait de "sacro-sainte recherche »! J'ai toujours eu l'idée qu'il fallait faire quelque chose en psychologie des religions, quelque chose qui n'existait pas, et j'ai toujours été très soutenu, y compris par mon ostrogoth de Robert Pagès dont j'ai fait l'éloge funèbre il y a quelques années ${ }^{6}$. Donc voilà un développement universitaire normal avec ma retraite prise il y a douze ans.

Les « cinq doigts de la main » du GSR qui dirigeaient la revue se réunissaient une fois par mois dans le bureau magistral de Desroche, chez lui-même. C'était un groupe solidaire mais où en même temps chacun s'opposait à l'autre. Danièle (Hervieu-Léger, ndr) disait : " ces gens-là se haïssent cordialement ». C'était peutêtre un peu exagéré, mais il y avait de cela. Il y avait donc là Desroche (qui présidait avec maestria), Poulat, Isambert (avec qui je n'ai pas été très lié), Maître (avec qui je m'entendais parfaitement) et le nouveau venu Séguy (hiératique, jésuite jusqu'au bout des ongles, un type extraordinaire. Quand j'allais au restaurant avec lui, il lui arrivait de demander qu'on lui fasse des nouilles à l'eau ! Et quand il nous recevait le dimanche après-midi, à Boulogne, le clou des festivités était le roudoudou, une décoction de calice de roses !). Dans ces réunions du comité de la revue, l'opposition entre Séguy et Maître était constante. Pour deux raisons : d'abord parce que Maître était communiste et ensuite parce qu'il mettait la religion en chiffres. Et donc, on m'a demandé de participer à ce comité, ça devait être à partir de 1969, pour m'occuper des tables analytiques puis du bulletin bibliographique. J’y suis resté vingt-trois ans. Dans cette période une chose m'a

4. Compte rendu d'Émile Poulat dans Archives de sociologie des religions, 1969, 27, p. 174-175. On signalera en outre le compte rendu de Jacques Maître sur la thèse de théologie de J.-P. D. soutenue quelques années auparavant à l'université catholique de Lille ("L'idée de Dieu chez les garçons de 7 à 16 ans. Contribution à l'étude expérimentale des idées religieuses "), Archives de sociologie des religions, 964, 17, p. 173-174.

5. Jean Maisonneuve, titulaire de la première chaire de psychologie sociale en 1966 à l'Université de Paris-Nanterre, est un auteur classique de cette branche disciplinaire. Ses ouvrages portent notamment sur les affinités, la dynamique de groupe, les représentations du corps.

6. J.-P. Deconchy, "Robert Pagès, un libertaire libérateur ", Psychologie française, 2007, 52-3, p. 257-263. 
frappé, c'était cette tendance à la diversification des disciplines et des terrains de recherche, sans grand effort de réflexion théorique. J'ai retrouvé cela lors du cinquantenaire de la revue en 2006, quand il y a eu une assemblée générale à l'IRESCO ${ }^{7}$. J'ai d'ailleurs été interviewé pour un film rétrospectif sur les Archives ${ }^{8}$. Sans aucune animosité de ma part, je constate d'ailleurs que sur les trois heures d'entretien, en est restée au montage une petite phrase de moi qui tournait à la gloire de Poulat ! Et au cours de ces vingt-trois ans, on m'a demandé d'associer ma petite équipe de psychologie sociale de la Sorbonne à l'édition de la revue.

Donc pour revenir à mon lien avec le GSR, il constituait, à côté du laboratoire de psychologie sociale, un point d'ancrage intellectuel inestimable. Les réunions mensuelles de la revue où je me trouvais le plus jeune et le plus respectueux aussi, m'ouvraient à des horizons sans cesse renouvelés. J'y étais en même temps la petite main chargée de tâches pratiques qui rebutaient chacun, comme la confection des tables analytiques avec Marie-Louise Letendre, une personne remarquable à tous égards. Je me souviens que j'allais rue d'Athènes répertorier les références qui étaient enregistrées sur des milliers de fiches entassées dans des cartons à chaussures qui s'empilaient dans le grenier. Je me suis ensuite occupé du bulletin des thèses initialement répertoriées par Poulat. Je me souviens que Desroche est intervenu pour que Poulat ne cosigne pas ce bulletin avec moi, car j'en avais pris toute la responsabilité. Ensuite, on m'a demandé de prendre la direction du bulletin bibliographique. Outre cette part de travail, j'ai quand même publié pas mal d'articles et de comptes rendus dans la revue, ce qui m'a beaucoup aidé dans mes réflexions. Le bulletin bibliographique a toujours été une grande affaire dans cette revue. Cette visée encyclopédique résultait du Trend Report de 1956 sur la sociologie des religions. Malgré l'absence de consensus théorique ou méthodologique entre les membres du comité de lecture, cet engagement fort dans le bulletin bibliographique avait quelque chose d'une théorie de la recension partagée par chacun. Lorsque Poulat dirigeait ce bulletin, il était impitoyable ; il relisait et corrigeait scrupuleusement chaque compte rendu. Si Desroche m'a appris à parler, Poulat m'a appris la grammaire. Au passage, Desroche était un génie, quelqu'un qu'on peut difficilement cerner.

Pour ce bulletin, il y avait d'abord les ouvrages qui arrivaient spontanément, et ceux que l'on commandait, chacun selon son axe de recherche. Ça m'a valu une belle bibliothèque qui me reste encore aujourd'hui. Donc pour ce bulletin, il n'y avait pas de doctrine mais une stratégie tendant à faire état des nouveautés et en même temps d'argumenter leur apport de connaissances. C'est là où j'en reviens à cette histoire de différenciation croissante des thématiques du GSR.

7. Voir «Les Archives... cinquante ans après ", Archives de sciences sociales des religions, 2006, 136.

8. Documentaire en ligne sur le site de la revue : assr.revues.org 
Aujourd'hui, on est frappé par le côté éclectique du contenu, ce qui n'est pas une critique, mais le constat d'une évolution du champ de recherche. Et dans le bulletin, on retrouve aujourd'hui également une certaine disparité entre les comptes rendus. Je pense parfois à certains d'entre eux comme ceux de Daniel Vidal qui sont intéressants mais souvent longs. Et je dois dire qu'à l'origine, la confection de ce bulletin se discutait en comité et chacun y participait activement. On était tous très concernés par cette affaire. Je me demande d'ailleurs si la pérennité de la revue n'est pas due en partie à cette structuration bibliographique. Je dois dire par parenthèse mon admiration pour la poursuite de ce travail avec la sortie de quatre numéros par an, ce qui n'est pas rien, j'imagine.

Pour revenir à mon trajet : j'ai quitté le groupe au début des années 1970 . Ceci dit je suis resté un des plus fidèles puisque par exemple j'assiste à toutes les obsèques. Jusqu'alors, c'est Poulat qui m'avertissait d'un décès. Alors pourquoi suis-je parti ? Il n'y avait dans ce mouvement aucune opposition ou rancœur à l'égard du groupe. Loin de là. Tout simplement, j'ai fondé un laboratoire de psychologie sociale à Nanterre qui a pris beaucoup de mon temps à l'époque. J'ai quand même mené à bien plus d'une quarantaine de thèses. Dans un domaine expérimentaliste qui exige beaucoup de temps. Donc, n'arrivant plus à suivre au GSR, je suis parti. Il faut dire que la vie d'un expérimentaliste ce n'est pas la même chose que celle d'un sociologue. Le temps de laboratoire dans le premier cas est essentiel. Écrire des livres n'est pas le but comme c'est le cas de la sociologie ou de l'histoire.

Ces disciplines littéraires sont par ailleurs ouvertes à la vie des institutions, de la cité comme on dit. Je ne dirai pas la fascination secrète du GSR pour l'Église catholique jusqu'à en épouser dans la pratique des relations interpersonnelles toutes les normes et les modes de faire. La hantise des fondateurs était qu'on les croie antireligieux. Finalement, Poulat a fait dehors l'œuvre qu'il aurait rêvé faire dedans. Il n'a jamais pris ses distances avec l'Église. Je me souviens maintenant qu'un jour, peu avant que je quitte le groupe, Poulat avait rassemblé l'équipe pour nous présenter Mgr Etchegaray ; je n'ai jamais compris pourquoi. Il s'agissait, à côté de la recherche de précieux garants comme Jean-Pierre Vernant, de faire indirectement obédience à l'autre bord. Je me demande si cette petite réunion n'a pas précipité les choses en moi pour m’éloigner du groupe. Ce qui m'a un peu étonné, c'est que mes livres et plus généralement les questions de psychologie sociale ont été par la suite peu présents dans la revue. Je me souviens d'un compte rendu de Paul Ladrière, un type sculpté dans l'angoisse, qui avait fait un gros effort pour recenser ma thèse d'État sur les sciences humaines et l'orthodoxie religieuse ${ }^{9}$. Mon livre sur les " animaux surnaturés » (2000) où je montre expérimentalement comment les représentations de l'homme vont à

9. P. Ladrière, "Raison et orthodoxie religieuse ", Archives de sciences sociales des religions, 1981, 51-2, p. 169-172. 
l'encontre des déterminismes de nature n'a pas été recensé. Lors des funérailles de Séguy, j'en ai touché un mot à Poulat qui m'a aussitôt répondu de lui envoyer l'ouvrage. Et ce phénomène, dans un long développement autour d'Henri Bremond, a alors trouvé le moyen de citer mon ouvrage en quelques mots furtifs.

$\mathrm{Au}$ fond, je crois que le groupe n'a jamais su intégrer la psychologie sociale à l'étude des religions. Certes il voulait de la psychologie mais à la manière d'Ignace Meyerson, un type extraordinaire mais qui était loin des perspectives expérimentales. Si j'avais fait de la psychologie sociale à la manière de Moscovici, je crois que ça aurait plus intéressé le milieu. Je crois que pour le groupe, je faisais plutôt des bricoles amusantes.

Il reste que par la suite, j'ai continué à fréquenter les uns et les autres. Séguy est venu chez moi à l'époque où mon épouse y était encore, et même par la suite quand elle m'a quitté et que je me suis retrouvé seul à élever deux jeunes enfants. Ce départ de mon épouse et ce moment difficile avec en plus la nouvelle chaire à Nanterre, tout cela a joué aussi dans mon départ du GSR. J'ai également entretenu quelques relations épisodiques avec Maître, mais sur la fin il ne m'a plus donné de ses nouvelles. Son parcours a quelque chose d'une épure : son idéologie marxiste de base ayant rencontré le démenti de l'histoire, il a trouvé une porte de sortie dans la psychologie clinique. Donc voilà quelques éléments de mon parcours avec le GSR.

Et lorsque vous l'avez quitté, le conflit entre Poulat et Isambert autour de la création du groupe de sociologie de l'éthique était consommé?

Je me souviens peu de cette tension, il y en a eu tellement ! À propos d'Isambert, je pense au conflit qui l'a opposé à Séguy également candidat à la chaire de l'EPHE (VI ${ }^{\mathrm{e}}$ section $)^{10}$. Ça reste entre nous, mais je pense que sur le fond Séguy était plus passionnant. Les travaux d'Isambert avaient moins d'impact à mon avis que ceux de Séguy qui en parallèle de Desroche passionnait l'auditoire. C'était en même temps une personnalité à la fois attachante et impossible à vivre. Je me souviens d'un débat sur la couleur des couvertures de la revue; lorsque quelqu'un a dit que le rouge serait pas mal, Séguy est alors monté sur ses grands chevaux pour dire qu'il démissionnerait si la couleur rouge était retenue. Alors pour revenir à l'histoire d'Isambert contre Poulat, je crois que l'association à la revue de ma petite équipe de psychologie sociale de 1979 à 1991 avait dû se faire un peu contre Isambert, de sorte que son groupe de sociologie de l'éthique figure comme une équipe aussi légère que la mienne. Autre type d'accroche qui me revient maintenant en parlant, c'est celui des liens entre le GSR et l'Université de Sherbrooke au Québec. Une affaire que Desroche avait lancée et que j'ai

10. Section de «Sciences économiques et sociales » créée en 1947 et devenue en 1975 l'EHESS. 
poursuivie au-delà de lui. Ça a été pour moi l'occasion de réaliser des manipulations intéressantes, notamment auprès des théologiens avec qui nous travaillions sur les fausses vérités. J'ai même écrit une fausse Bible pour les besoins de l'expérimentation. Il s'agissait par exemple de démontrer que chez les plus orthodoxes la stratégie n'était pas de donner une information nouvelle mais de conforter une information acquise. Des choses que je n'aurais jamais pu faire en France. Séguy n'aimait pas du tout ce genre de manipulation. Le comble de l'affaire de la fausse Bible, c'est que certains clercs que j'avais rencontrés là-bas ont quand même utilisé le texte falsifié pour les besoins de la pastorale car ils le trouvaient plus beau que les versions autorisées !

Donc vous avez quitté le groupe en 1991, tout en entretenant quelques relations avec les uns ou les autres, mais plus généralement qu'est-ce qu'il en est de la psychologie des religions? Pourquoi cette spécialité est-elle en France si peu développée?

J'ai longtemps cru que c'était un drame, mais avec le temps j'ai compris que c'était normal. Si l'on exclut la partie clinicienne et analytique de la psychologie qui a pris de l'ampleur à partir de la thématique religieuse, j'en suis venu à l'idée que la psychologie expérimentale des religions n'avait pas de raison d'être en tant que spécialité, car c'est seulement un terrain d'expérimentation parmi d'autres. Dès qu'on théorise la notion de religion dans une perspective cognitiviste on se trouve en face des questions communes à toute idéologie. On retrouve le vieux principe aristotélicien du genre prochain, c'est-à-dire de l'idéologie. Mais en même temps, si on ne compte plus les travaux sur la sociologie de l'idéologie, en matière de psychologie de l'idéologie, il n'y a pas grand-chose. Si je mets à part le pôle du langage, vous avez en psychologie sociale une entrée par les représentations sociales telles que Moscovici les avait définies avec une sensibilité nettement sociologique. Mais vous avez aussi une entrée plus proprement psychologique avec une nouvelle dichotomie : la distinction entre le clinique et l'expérimental. Dans la partie clinique, vous avez évidemment la psychanalyse qui en matière de religion a fait beaucoup jusqu'à être un substitut de la théologie. Mais dans la partie expérimentale, lorsqu'on a fait la synthèse artificielle de l'objet, on n'en reste qu'à quelques hypothèses par nature falsifiables. Nous sommes dès lors très peu à travailler sur la paillasse, avec des entreprises coûteuses, pas seulement en argent mais en termes de protocoles méthodologiques. De plus, en ce qui concerne la thématique religieuse, elle est principalement liée en sciences humaines à des chercheurs qui pour la plupart ont fait l'expérience personnelle du religieux. Ce qui n'est pas le cas des psychologues expérimentaux.

Comment expliquez-vous la résistance des membres du GSR à la perspective de la psychologie expérimentale?

Il faut dire que les fondateurs marquants du groupe, qui étaient par ailleurs mes aînés, trouvaient que je m'amusais plus que je cherchais vraiment quelque 
chose de tangible. Notre savoir qu'on peut dire prudentiel et conditionnel n'apporte pas non plus beaucoup d'énoncés qui ont à voir avec la signification du monde ou de l'action sociale. Il ne peut être perçu que secondaire par rapport au discours à dimension politique. Les travaux actuels des cognitivistes comme Pascal Boyer ou Scott Atran qui prennent la religion comme objet, et qui ont un certain succès, ne s'appuient pas sur une perspective expérimentale. Et à Nanterre, depuis mon départ du GSR, je n'ai vraiment pas poussé la thématique religieuse. Finalement comme je vous l'ai dit, celle-ci est hors sujet en matière de psychologie cognitive et expérimentale. La question du traitement de l'information comme vraie malgré son absence de fondement empirique est une thématique transversale qui dépasse de loin l'objet religieux. Nous avons par exemple récemment travaillé sur les représentations photographiques de la destruction des tours de New York en 2001. Le visage du démon apparaît à un moment dans la forme de la fumée. Le travail consistait à montrer comment cette forme prend sens aux yeux des observateurs. Mon idée plus générale c'est que le religieux n'est qu'une base pour l'étude des mécanismes cognitifs, notamment le contrôle de la rationalité. Lorsque la rationalité d'un énoncé montre des signes de faiblesse, le groupe social qui l'émet tente de la renforcer. Sans contrôle social, l'irrationalité est manifeste.

Et donc pour finir, j'aimerais revenir encore sur cette histoire de différenciation thématique et disciplinaire du groupe initial. Cet après-midi d'études pour le cinquantenaire de la revue était passionnant, par le film, par les contributions des uns et des autres. Mais j'avais un peu l'impression qu'il s'agissait d'étendre toujours l'objet religieux dans ses multiples formes dans l'espace et dans le temps plutôt que de se demander s'il avait une consistance propre. La question de la réduction du multiple à quelques opérateurs identifiables, les plus unificateurs possible des phénomènes les plus divers, cette question normale en toute science semblait ici complètement évacuée.

Il y avait là quelque chose de la sacralisation du multiple. Une arborescence à peine réticulée. Je dois dire que depuis l'origine ce processus extensif était en marche. Avec en plus l'idée d'être utile y compris aux institutions religieuses. Ce que j’ai été amené à récuser à plusieurs reprises. Position critique qui m'a valu un jour des remarques étranges du genre de celle d'Isambert qui me reprochait d'attaquer Ladrière. Chose à laquelle je n'avais absolument pas pensé...

Si l'on se penche sur quelques trajectoires, on constate cependant que l'objectif de trouver un opérateur universel à la multiplicité des faits religieux reste bien présent. Danièle Hervieu-Léger par exemple l'a trouvé en inscrivant pratiques et croyances dans une lignée qui fasse autorité et société.

Oui tout à fait, vous avez raison, et tout cela avec une virtuosité de langage exceptionnelle. Un charisme qui ressemble beaucoup à celui de Desroche. Ce sont des êtres extrêmement inventifs qui introduisent de nouveaux concepts en 
parlant tout haut. Mais l'une comme l'autre n'étaient pas des expérimentateurs proprement dits. Et pour un type comme Poulat, essayer d'introduire une construction théorique revenait à faire de l'idéologie. Maître aussi dans sa première mouture. Il s'agissait d'enregistrer et de classer des données, et ceci à l'infini. Le monde religieux est inépuisable. Voilà donc quelques souvenirs et réflexions que j'ai eu plaisir à évoquer avec vous...

\section{Bibliographie}

Structure génétique de l'idée de Dieu chez les catholiques français, 1967, Bruxelles, Éditions Lumen Vitae, 237 p.

L'orthodoxie religieuse. Essai de logique psycho-sociale, 1971, Paris, Éditions ouvrières, $373 \mathrm{p}$.

Orthodoxie religieuse et sciences humaines, 1980, Paris-La Haye, Mouton, 340 p.

Psychologie sociale. Croyances et idéologies, 1989, Paris, Méridiens-Klincksieck, 280 p.

Les animaux surnaturés. La construction mentale de la singularité bumaine, 2002, Grenoble, Presses universitaires de Grenoble, 187 p.

L'autoritarisme (avec Vincent Dru), 2007, Grenoble, Presses universitaires de Grenoble, $107 \mathrm{p}$.

Living in an Asymmetrical World. How Writing Direction Affects Thought and Action (avec Anne Maas et Caterina Suitner), New York, Psychology Press, 2014, 170 p. 